Andreas Jacobsen

Intelligenz von Unternehmen 


\section{DVV Wirtschaftswissenschaft}


Andreas Jacobsen

\section{Intelligenz von Unternehmen}

Management technologischer

Kompetenz in Innovationsprozessen

Mit einem Geleitwort von Prof. Dr. Heiner Müller-Merbach

Springer Fachmedien Wiesbaden $\mathrm{GmbH}$ 
Die Deutsche Bibliothek - CIP-Einheitsaufnahme

\section{Jacobsen, Andreas:}

Intelligenz von Unternehmen : Management technologischer Kompetenz

in Innovationsprozessen / Andreas Jacobsen. Mit einem Geleitw. von

Heiner Müller-Merbach. - 1. Aufl.

(DUV: Wirtschaftswissenschaft)

Zugl.: Kaiserslautern, Univ., Diss., 1999

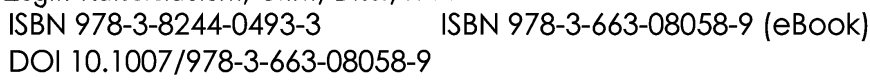

Vom Fachbereich Sozial- und Wirtschaftswissenschaften der Universität Kaiserslautern genehmigte Dissertation.

D 386

1999

1. Auflage November 2000

Alle Rechte vorbehalten

(C) Springer Fachmedien Wiesbaden 2000

Ursprünglich erschienen bei Deutscher Universitäts-Verlag GmbH, Wiesbaden, 2000

Lektorat: Ute Wrasmann / Monika Mülhausen

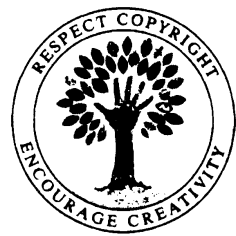

Das Werk einschließlich aller seiner Teile ist urheberrechtlich geschützt. Jede Verwerłung außerhalb der engen Grenzen des Urheberrechtsgesetzes ist ohne Zustimmung des Verlages unzulässig und strafbar. Das gilt insbesondere für Vervielfältigungen, Ubersetzungen, Mikroverfilmungen und die Einspeicherung und Verarbeitung in elektronischen Systemen.

www.duv.de

Höchste inhaltliche und technische Qualität unserer Produkte ist unser Ziel. Bei der Produktion und Verbreitung unserer Bücher wollen wir die Umwelt schonen. Dieses Buch ist auf säurefreiem und chlorfrei gebleichtem Papier gedruckt. Die Einschweißfolie besteht aus Polyäthylen und damit aus organischen Grundstoffen, die weder bei der Herstellung noch bei der Verbrennung Schadstoffe freisetzen.

Die Wiedergabe von Gebrauchsnamen, Handelsnamen, Warenbezeichnungen usw. in diesem Werk berechtigt auch ohne besondere Kennzeichnung nicht zu der Annahme, dass solche Namen im Sinne der Warenzeichen- und Markenschutz-Gesetzgebung als frei zu betrachten wären und daher von jedermann benutzt werden dürften. 
Carl Wilhelm,

Sophie Johanna,

Paulina Herta,

Marie Helga

und Dagmar 


\section{Zum Geleit}

Wie intelligent ist dieser Mensch? Mit welcher Intelligenz agiert die Unternehmung XY auf dem Markt? Mit welcher Intelligenz tritt eine Nation in den Wettstreit mit den anderen? Intelligenz als Inbegriff der Wettbewerbsfähigkeit!?

Eignet sich "Intelligenz" nicht als zentraler Erfolgsfaktor von Individuen und Institutionen schlechthin? Kaum ein anderer Begriff bringt die Erfolgsdeterminanten zielschärfer auf einen einzigen Nenner. Mag man Intelligenz auch noch so unterschiedlich definieren, so scheinen die Vorstellungen vom eigentlichen Kern der Intelligenz in hohem Maße deckungsgleich zu sein: als Quelle der Kraft, Probleme lösen zu können, als Wurzel der Wettbewerbsähigkeit.

In allen Regionen der Triade - Europa, Nordamerika, Südostasien - sind Managementlehren entstanden, in denen die Intelligenz eine zentrale inhaltliche wie auch begriffliche Rolle spielt: "Organizational Intelligence" oder "Organisatorische Intelligenz", „Intelligenz der Unternehmung“ oder "Intelligente Unternehmung“, „Intelligent Enterprise“, „Business Intelligence", "L'intélligence économique" etc., alles Titel von Veröffentlichungen der 1990er Jahre. Hinter den Titeln stehen Versuche, durch Entfaltung und Nutzung der individuellen und kollektiven Intelligenz die Wettbewerbskraft von Unternehmungen und anderen Institutionen zu stärken.

An die bisherigen Ansätze, kollektive Intelligenz als Zentrum der Wettbewerbskraft zu sehen, knüpft Dr. Jacobsen mit diesem Buch an. Er betrachtet denjenigen Funktionsbereich der Unternehmungen, in dem die Bedeutung der Intelligenz am offensichtlichsten und stärksten sein mag: Forschung und Entwicklung.

Dr. Jacobsen nimmt Bezug auf die psychologische Intelligenzforschung und bereichert mit ihr seine Sicht der intelligenzorientierten Managementlehre. Das ist vielversprechend. Bewußtes auf Intelligenz bezogenes Führen erfordert im Grundsatz einen gewissen inneren Abstand vom Tagesgeschäft: geistigen Freiraum für das Reflektieren über die "Intelligenz" der Institutionen, in die man selbst eingebunden ist: Wie intelligent ist das politische Gremium, in dem ich mitwirke? Wie intelligent ist meine Unternehmung, mein Unternehmensbereich, meine Abteilung? Wie intelligent ist meine Nation? Durch Reflektieren über Intelligenz vertieft sich das Bewußtsein von Intelligenz. Zu solcher Bewußtseinsvertiefung regt Dr. Jacobsen an. 
"Organizational Intelligence" als Orientierungspunkt einer neuen Managementlehre soll ein Umdenken bewirken: Alle Tätigkeiten der Unternehmung werden dabei als Ergebnis einer - höheren oder niedrigeren - Intelligenz gesehen, d.h. als durch menschlichen Geist bewirkt. Dadurch wird weder die Betriebswirtschaftslehre auf den Kopf gestellt, noch ist eine völlig neue Managementpraxis zu erwarten. Vielmehr geht es um einen Wandel der Sichtweise, um eine etwas andere Sichtweise von dem Geschehen in der Unternehmung; und dieser Wandel der Sichtweise wird Wirkung zeigen und am Erfolg der Unternehmung sichtbar werden.

Prof. Dr. Heiner Müller-Merbach Universität Kaiserslautern 


\section{Vorwort}

Intelligenz von Unternehmen: Was nun mit der Veröffentlichung dieses Buches seinen Abschluß findet, ging bis dahin einen weiten Weg der Recherche und Reflektion, des eigenen Lernens und der Diskussion mit anderen, des anregenden Lesens und des teilweise anstrengenden - Schreibens. Es begann mit dem Interesse für ein spannendes und vielschichtiges Thema, welches Prof. Dr. Heiner Müller-Merbach im Geleitwort zu diesem Buch als „zentralen Erfolgsfaktor" charakterisiert.

Keine Frage: Auch die vorliegende Arbeit hat ihre eigenen, „zentralen Erfolgsfaktoren“: Mein akademischer Lehrer, Prof. Dr. Heiner Müller-Merbach, hat dieser Arbeit durch seine offene und klare Denkweise wertvolle Impulse gegeben. Hierür und für unsere langjährige Zusammenarbeit, deren Bedeutung für mich weit über das vorliegende Buch hinausreicht, danke ich inm sehr herzlich. Ebenfalls bedanke ich mich bei der Stiftung Rheinland Pfalz für Innovation, die diese Arbeit gefördert hat.

Als „zentraler Erfolgsfaktor" erwies sich auch die Zusammenarbeit mit meinen ehemaligen Kollegen am Lehrstuhl von Prof. Müller-Merbach, die durch konstruktive Diskussion, Hilfsbereitschaft und Freundschaft geprägt war. Dafür danke ich Klaus Gesmann, Christian Guhl, Dr. Roland Hanebeck, Rainer Kellerhals, Dr. Birgid Kränzle, Dr. Guido Krupinski, Volker Kurz, Peter Lebesmühlbacher, Anneliese Leipold, Dr. David Meyer, Prof. Dr. Martin Möhrle, Dr. Christian Momm, Dagmar Schwarzer, Dr. Jens Stief, Dr. Christoph Vogel und Vera Wolf.

Durch Unterstützung bei der Erstellung der Druckvorlage und treffsicheres Aufspüren von Druckfehlern hat Frau Herta Jacobsen zum Gelingen dieser Arbeit beigetragen. Hierfür danke ich ihr herzlich.

Schließlich gehört zu den „zentralen Erfolgsfaktoren“ meine eigene Familie: Für den Verzicht auf gemeinsame Zeit, für ihr Verständnis und für erholsame Ablenkung danke ich meiner Frau und meinen Kindern ganz herzlich. Sie haben nicht nur zum Fortschritt dieser Arbeit beigetragen, sondern stets auch meinen Blick auf die neben solcher Abeit „wirklich wichtigen“ Dinge des Lebens gelenkt. 


\section{Inhaltsverzeichnis}

Zum Geleit

Vorwort

IX

1 Informationsgesellschaft und Wettbewerbsfähigkeit von Unternehmen 1

2 Unternehmensintelligenz: Fähigkeit zur Kompetenzentwicklung ....................... 7

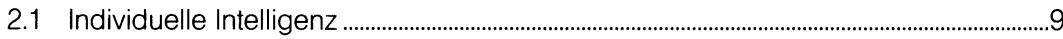

2.1.1 Intelligenz als Konzept.................................................................................................10

2.1.2 Drei Aspekte menschlicher Intelligenz: Funktionen, Struktur, Inhalt..............11

2.2 Unternehmensintelligenz: Intelligenz des Unternehmens vs. Intelligenz im Unternehmen....................................................................................................................................... 14

2.2.1 Unternehmen als eigenständiges Subjekt ............................................................15

2.2.2 Unternehmensintelligenz als Intelligenz des Unternehmens.............................16

2.3 Bestehende Ansätze zur Unternehmensintelligenz...........................................................17

2.3.1 Ansätze zur Intelligenz von Unternehmen oder Organisationen im Überblick.

2.3.2 Wilensky: „Organizational Intelligence“ und „Intelligence failures“................20

2.3.3 Scheuss: „Institutionelle Intelligenz" als Kapazität zur Informationsverarbeitung

2.3.4 Matsuda: „Organizational Intelligence“ als Prozeß und als Produkt .............22

2.3.5 Müller-Merbach und Momm: Intelligenz der Unternehmung als Gesamtheit von Information, Wissen und Meinung.

2.3.6 Oberschulte: Organisatorische Intelligenz als System.

2.3.7 Weitere Ansätze zur Intelligenz von Unternehmen oder Organisationen.

2.3.8 Zusammenfassender Vergleich der Ansätze: Inhalt, Funktionen und Struktur der Intelligenz von Unternehmen oder Organisationen.

2.4 Ein Modell der Unternehmensintelligenz..................................................................................28

2.5 Kompetenz eines Unternehmens ..............................................................................................32

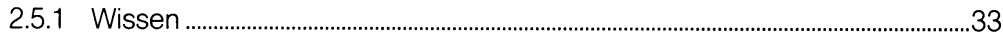

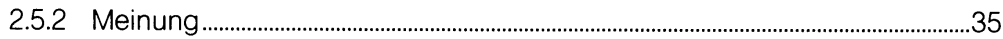

2.5.3 Information.....................................................................................................................36

2.5.4 Kompetenz als Gesamtzusammenhang von Wissen, Meinung und Information. 
3 Gestaltung der Unternehmensintelligenz...............................................................41

3.1 Entwicklung der Funktionen der Unternehmensintelligenz ............................................43

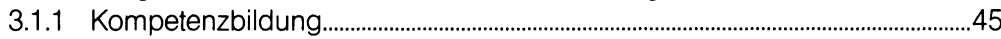

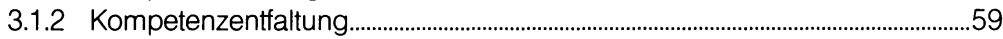

3.2 Gestaltung einer Struktur der Unternehmensintelligenz....................................................68

3.2.1 Verankerung der Kompetenzentwicklung in der Unternehmensorganisation.........................................................................................70

3.2.2 Ausrichtung der Unternehmenskultur auf Kompetenzentwicklung ................76

3.2.3 Nutzung von IKT-Systemen für die Kompetenzentwicklung .............................82

3.3 Agenda für die Gestaltung der Unternehmensintelligenz .................................................90

4 Nutzung der Unternehmensintelligenz für betriebliche Innovationen...............93

4.1 Innovationen durch die Erneuerung von Produkten und

Produktionsverfahren .................................................................................................................95

4.1.1 Innovation: Neue Produkte und Produktionsverfahren ........................................96

4.1.2 Innovationsprozeß: Ablauf der Entwicklung und Einführung von Produkten und Produktionsverfahren .....................................................................101

4.1.3 Innovationsstruktur: Verbund der betrieblichen Funktionsbereiche............110

4.2 Ausrichtung der Unternehmensintelligenz auf betriebliche Innovationen..............117

4.2.1 Technologische Kompetenz: Technologie, Innovationsbewußtsein und F\&E-Information

4.2.2 Ausrichtung von Funktionen und Struktur der Unternehmensintelligenz.

5 Bildung und Entfaltung technologischer Kompetenz.

5.1 Bildung technologischer Kompetenz ……......................................................................130

5.1.1 Wahrnehmung: Technologische Frühaufklärung ..............................................133

5.1.2 Lernen: Technologieenwerb............................................................................................. 138

5.1.3 Anwendung: Einführung von Produkten und Produktionsverfahren ..........151

5.2 Entfaltung technologischer Kompetenz.................................................................................166

5.2.1 Kommunikation: Innerbetrieblicher Transfer technologischer Kompetenz.

5.2.2 Speicherung: Bewahrung technologischer Kompetenz in einem Unternehmen 


\section{Gestaltung der Rahmenbedingungen für die Bildung und Entfaltung} technologischer Kompetenz.

6.1 Organisation der Bildung und Entfaltung technologischer Kompetenz...................184

6.1.1 Gestaltung der Aufbauorganisation ..........................................................................184

6.1.2 Gestaltung der Ablauforganisation..............................................................................190

6.2 Förderung einer kommunikations- und innovationsfreundlichen

Unternehmenskultur .................................................................................................................198

6.2.1 Anforderungen an die Unternehmenskultur ...........................................................199

6.2.2 Veränderung einer Unternehmenskultur.......................................................................203

6.3 Einführung von Informations- und Kommunikationssystemen.....................................206

6.3.1 Informationsstrukturierung mit Hilfe des Objekttypenansatzes .....................207

6.3.2 Inhaltlicher Aufbau der IKT-Systeme...........................................................................210

6.3.3 Nutzung unterschiedlicher Informations- und

Kommunikationstechnologien..........................................................................................223

7 Zusammenfassung................................................................................................227

Literaturverzeichnis..................................................................................................229 